\title{
Communication
}

\section{Gravitational Lensing of Supernova Neutrino Bursts}

\author{
John M. LoSecco
}

check for updates

Citation: LoSecco, J.M. Gravitational Lensing of Supernova Neutrino Bursts. Universe 2021, 7, 335. https:// doi.org/10.3390/universe7090335

Academic Editor: Lorenzo Iorio

Received: 30 July 2021

Accepted: 5 September 2021

Published: 8 September 2021

Publisher's Note: MDPI stays neutral with regard to jurisdictional claims in published maps and institutional affiliations.

Copyright: (c) 2021 by the authors. Licensee MDPI, Basel, Switzerland. This article is an open access article distributed under the terms and conditions of the Creative Commons Attribution (CC BY) license (https:// creativecommons.org/licenses/by/ $4.0 /)$.
Physics Department, University of Notre Dame du Lac, Notre Dame, IN 46556-5670, USA; losecco@nd.edu

\begin{abstract}
Supernova neutrino bursts have been observed from extragalactic distances. This note addresses the question of how gravitational lensing could distort the information in the burst. We apply the gravitational lens hypothesis to try to understand the time and brightness structure of the SN1987A neutrino observations. Estimates of a possible lensing mass and alignment are made. These estimates suggest a path to verification.
\end{abstract}

Keywords: gravitational lens; neutrinos; SN1987A

\section{Introduction}

Observations of neutrinos from gravitational stellar collapse, a supernova, has the ability to peer into the heart of a transient critical event in the evolution of our universe. In a few seconds a mature massive star transforms into a neutron star or black hole releasing most of the binding energy as neutrinos. The subsequent explosion ejects the heavy elements into interstellar space and creates those beyond the nuclear binding energy peak. Observation of these neutrinos can tell us a great deal about the mechanism unless they are distorted en route to the detection.

Attenuation is considered unlikely since neutrinos have a very low rate of interaction with matter.

\section{Gravitational Effects}

Unlike particle-like interactions, which are considered unlikely, the neutrino interaction with gravitation is small but can accumulate over the flight time of the burst. The 1987A neutrino burst observations led to two verifications of the equivalence principle for neutrinos. The close arrival time between neutrinos and light [1,2] put bounds on possible violations of the equivalence principle, including the Shapiro delay [3] (the accumulated gravitational time delay due to propagation through a gravitational field and the added distance due to curvature), which is estimated at 4.8 months for both light and neutrinos. Angular analysis of the events suggests the presence of both neutrino and antineutrino events in the burst which constrains the difference in time delay between matter and antimatter to essentially the length of the burst, a few seconds. This neutrino/antineutrino comparison is a test of $\mathrm{CP}$ violation in general relativity $[4,5]$.

\section{Gravitational Lensing and Microlensing}

Gravitational lensing [6-9] embodies the gravitational induced curvature in both space and time. General relativity predicts that a ray of light with a distance of closest approach $r$ to a star of mass $M$ will be deflected toward the mass $M$ by the angle $v=\frac{4 G M}{c^{2} r}=\frac{K}{r}$. Multiple images and time delays are possible if multiple light rays from the same source are directed toward the observer as illustrated in Figure 1. An amplification of the source brightness can occur, as expected from optical lenses. In the case of microlensing, the images are not spatially resolved and the evidence may be in the form of variation in the brightness, $L_{1}+L_{2}$, as the relative positions of the source, the lens, and the observer change. Refsdal [7,9] and Leibes [8] have computed the brightness amplification and the time delay between the images. 


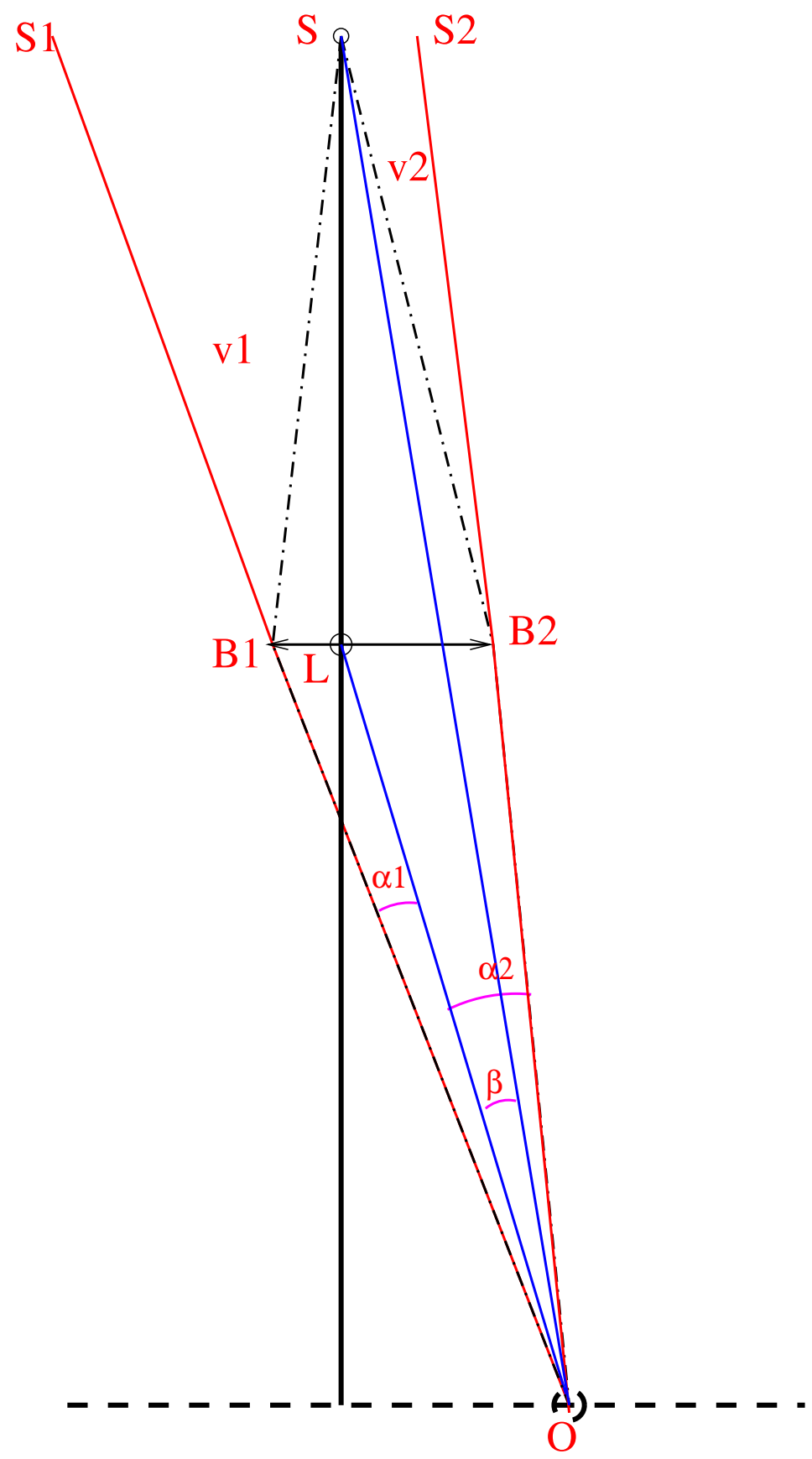

Figure 1. The geometry of a gravitational lens: The source at $S$ when viewed from $O$ appears at the locations $S_{1}$ and $S_{2}$ due to the deflections at $B_{1}$ and $B_{2}$ caused by the bending of the light by the lens at $L$. The angle $S O L$ is $\beta$ and $S_{1} O S_{2}$ is the angle between the two images $\alpha=\alpha_{1}+\alpha_{2}$.

One restriction imposed by Refsdal [7,9] that can be dropped is that for neutrinos, in most cases, the distance of closest approach to the lens mass, $r$, can be smaller than the radius of the lens with a suitable redefinition of $M$, since there will be little attenuation of the neutrino flux.

Figure 1 illustrates the lens geometry and defines many of the variables. For a pair of images one finds the brightness of the images, $L_{1}$ and $L_{2}$,

$$
\begin{aligned}
L_{1} & =\frac{1}{4}\left(2+\frac{\alpha}{\beta}+\frac{\beta}{\alpha}\right) L_{N} \\
L_{2} & =\frac{1}{4}\left(-2+\frac{\alpha}{\beta}+\frac{\beta}{\alpha}\right) L_{N}
\end{aligned}
$$


where $L_{N}$ is the brightness in the absence of a lens.

The time delay is approximately

$$
\begin{gathered}
\Delta t \approx n D_{L} \alpha \beta c^{-1}\left(1-\frac{1}{3} \frac{\beta^{2}}{\alpha^{2}}\right) \approx n D_{L} \alpha \beta c^{-1} \approx n D_{L} \alpha_{0} \beta c^{-1} \\
\Delta t \approx \frac{16 G}{c^{3}} \frac{\beta}{\alpha_{0}} M=\frac{8 G}{c^{3}} \frac{\beta}{\theta_{E}} M
\end{gathered}
$$

where

$$
\begin{gathered}
\alpha=\sqrt{\alpha_{0}^{2}+\beta^{2}} \approx \alpha_{0}\left(1+\frac{1}{2} \frac{\beta^{2}}{\alpha_{0}^{2}}\right) \approx \alpha_{0} \\
\alpha_{0}=\frac{4}{c} \frac{\sqrt{G M}}{\sqrt{n D_{L}}}
\end{gathered}
$$

and $\beta$ is the angular separation between the lensing object and the direction of the source, $\alpha$ is the angular separation of the two images, $D_{L}$ is the distance from the observer to the lens, $n=\frac{D_{S}}{\left(D_{S}-D_{L}\right)}$, the ratio of the distance from the source to the observer to the distance from the source to the lens. $n$ always appears in the product $n D_{L}=\frac{D_{S} D_{L}}{\left(D_{S}-D_{L}\right)}=\frac{D_{L}}{\left(D_{S}-D_{L}\right)} D_{S}$ which is the ratio of the observer to lens distance to the lens to source distance times the distance to the source. $\alpha_{0}$ is twice the value of the Einstein angle, $\theta_{E}$.

It is clear from the equation for $L_{2}$ that for the second image to be observable one needs $\beta \lesssim \alpha_{0}$.

$$
L_{2}=\frac{1}{4}\left(-2+\frac{\alpha}{\beta}+\frac{\beta}{\alpha}\right) L_{N} \rightarrow \lim _{\frac{\beta}{\alpha_{0}} \rightarrow \infty} \frac{1}{4}\left(-2+\left(1+\frac{1}{2}\left(\frac{\alpha_{0}^{2}}{\beta^{2}}\right)+1-\frac{1}{2}\left(\frac{\alpha_{0}^{2}}{\beta^{2}}\right)\right) L_{N} \rightarrow 0\right.
$$

and $L_{1} \rightarrow L_{N}$

The time difference between pairs of images, $\Delta t$, is proportional to the ratio $\beta / \alpha_{0}$ and to the lensing mass $M$.

If the two images cannot be resolved the observed brightness is the sum of $L_{1}$ and $L_{2}$.

$$
L_{1}+L_{2}=\frac{1}{2}\left(\frac{\alpha}{\beta}+\frac{\beta}{\alpha}\right) L_{N}
$$

It is also noteworthy that

$$
L_{1}-L_{2}=L_{N}
$$

and

$$
\frac{L_{1}+L_{2}}{L_{1}-L_{2}}=\frac{1}{2}\left(\frac{\alpha}{\beta}+\frac{\beta}{\alpha}\right)
$$

which is a function of only one variable $\frac{\alpha}{\beta}$ or $\frac{\alpha_{0}}{\beta}$.

From two images one can extract $\frac{\alpha_{0}}{\beta}$ from the brightness ratio $L_{2} / L_{1}$. Once $\frac{\alpha_{0}}{\beta}$ is known the lens mass can be extracted from the time delay between the images.

\section{Neutrinos from SN1987A}

The only supernova which has been observed in neutrinos was SN1987A on 23 February 1987. Four detectors reported observations of neutrinos on the same day the supernova light was first seen [10-16]. These observations are summarized in Table 1.

The raw information in the Table has not been corrected for the detection efficiency and energy and trigger bias. IMB, for example, could barely detect events below $20 \mathrm{MeV}$. The event rate in a neutrino detector is proportional to the interacting mass. In this case the interacting mass is almost exclusively the protons (hydrogen). The Kamiokande $[10,11]$ and IMB [12] detectors are composed of water with $11.2 \%$ of the mass as hydrogen. 
Baksan [13-15] and UNO [16] are composed of liquid scintillator with $14.4 \%$ of the mass as hydrogen.

The number of events recorded in detector $D$ is

$$
N_{D}=N_{A} M_{D} P_{D} \int F\left(E_{v}\right) \sigma\left(E_{v}\right) \epsilon_{D}\left(E_{v}\right) d E_{v}
$$

where $N_{A}$ is Avogadro's number, $M_{D}$ is the detector mass in grams, $P_{D}$ is the fraction of the mass in hydrogen, $\epsilon_{D}\left(E_{v}\right)$ is the energy dependent efficiency for the detector. $F\left(E_{v}\right)$ and $\sigma\left(E_{v}\right)$ are the neutrino flux and cross section which are the same for all detectors.

The initial time in the Table was synchronized with a time standard by UNO, IMB, and Baksan. Kamiokande used a computer clock set at computer boot from a watch with a quoted absolute accuracy of $\pm 60 \mathrm{~s}[10,11]$.

Table 1. Summary of the reported neutrino observations of SN1987A. The mean energy is a simple average of the energy of the reported events. It is not corrected for efficiency. The mean energy gives a rough idea of the portion of the energy spectrum sampled by that detector. The detectors have different capabilities and can only be compared after corrections for inefficiencies and other biases are accounted for.

\begin{tabular}{ccccccc}
\hline Name & $\begin{array}{c}\text { Mass } \\
\text { Metric Tons }\end{array}$ & $\begin{array}{c}\text { Time } \\
\text { UTC }\end{array}$ & $\begin{array}{c}\text { Duration } \\
\text { Seconds }\end{array}$ & $\begin{array}{c}\text { Number of } \\
\text { Events }\end{array}$ & $\begin{array}{c}\text { Mean Energy } \\
\text { MeV }\end{array}$ & $\begin{array}{c}\text { Energy Range } \\
\text { MeV }\end{array}$ \\
\hline UNO & 90 & $02: 52: 36.79$ & 7.01 & 5 & 8.4 & $7-11$ \\
\hline Kamiokande & 2140 & $07: 35: 35$ & 12.439 & $9+3=12$ & 14.7 & $6.3-35.4$ \\
\hline IMB & 5000 & $07: 35: 41.37$ & 5.59 & $6+2=8$ & 32.5 & $20-40$ \\
\hline Baksan & 200 & $07: 36: 11.818$ & 9.099 & $3+2=5$ & 18.1 & $12-23.3$ \\
\hline
\end{tabular}

\section{Observational Evidence}

Observational evidence for gravitational lensing of neutrino bursts is limited, since as of this writing only one burst has been observed. The four reported observations in Table 1 have many incompatibilities. It is unlikely that gravitational lensing would be able to explain them.

All of the observations were made from Earth. The neutrino source was in the Large Magellanic Cloud about $50 \mathrm{kpc}$ away. The maximum angular separation between the observations as viewed from the source is $8 \times 10^{-15}$ radians so all observations of the supernova neutrinos saw the same thing. Differences between the observations can occur because of differences in the sensitivity of each detector, instrumental effects and problems, or background events unrelated to the supernova. For example the IMB detector had a $14 \%$ efficiency for triggering the recording of an event at $20 \mathrm{MeV}$ and lower efficiency at lower energies. It is unlikely IMB could have seen the events reported by UNO, all below $11 \mathrm{MeV}$, even though IMB had a proton mass 43 times larger. Kamiokande spans the broadest energy range with a good sensitivity overlap with all detectors.

One should also be wary of overestimating the precision of the measurements. Neutrino observations are made quanta by quanta. One wants to understand the underlying distributions based on a limited number of samples. For example, the accuracy of time differences for events from the same detector is about 1 millisecond, but the rate of sampling, in places, is well below this, about one event per second. So time differences between images only have a physical significance to a second or more.

Lensing manifests itself in three ways. There can be multiple images. The images can be brighter than one which has not been lensed. The different images can have time delays between them due to the different paths taken to the observer. In the case of simply two images there are four observables, the angle $\alpha$ between the images, the brightness $L_{1}$ and $L_{2}$ of each image, and the time delay between the images.

The detection reaction for most of these events was electron antineutrino charged current with a target proton, producing a positron and a neutron, $\bar{v}_{e}+P \rightarrow e^{+}+N$. This 
reaction can provide good energy resolution, even in the absence of observing the neutron, but it does not provide any appreciable directionality. (Some experiments have tried to determine the direction of the neutrino by measuring the distance between the source of the positron and the neutron capture. Angular sensitivities of about $8^{\circ}$ are possible with very large samples [17]. None of the experiments in Table 1 used this method.) The lack of directional sensitivity makes the observation of the angle $\alpha$ between any two images unlikely.

The lens is geometrical so it is expected to be achromatic. It will not distort the neutrino spectrum by bending different neutrino energies to different angles. In the case of light, the images may have different spectra, since the paths through the interstellar medium will differ and the two paths may encounter different materials.

One can use the time distribution, such as time clustering, to identify gravitational lensing of the neutrino burst. Brightness differences between these clusters can also help identify a lensing event. If the time clusters have distinct spectra, except for brightness differences, it is less likely they manifest multiple observations of the same event. A timing difference can only be observed if both lensed images are bright enough to be observed.

The recorded times of the events, in particular the time of events from one detector relative to other events from the same detector, are well measured. In principle, the relative time of events from standard time synchronized experiments should also have good relative time measurements. Two timing issues are apparent from Table 1. UNO reported a signal $4 \mathrm{~h}$ and $43 \mathrm{~min}$ before IMB and Baksan. IMB reported a signal about $30.4 \mathrm{~s}$ before Baksan. The difference in nominal time of $6 \mathrm{~s}$ between Kamiokande and IMB has no significance given Kamiokande's \pm 1 min error on the absolute time. As mentioned above, except for instrumental differences, all detectors should see the same thing. Lensing can not explain the differences in start times.

The Kamiokande data has a $7.3 \mathrm{~s}$ gap starting at $1.915 \mathrm{~s}$, see Figure 2 . The full burst reported by Kamiokande has a duration of $12.439 \mathrm{~s}$. The data could be understood as two bursts. The first lasting $1.915 \mathrm{~s}$ contained nine events and the second lasting $3.22 \mathrm{~s}$ had three events. The time difference between these two pulses is $9.219 \mathrm{~s}$. Using the event counts as an estimate of the brightness suggests $L 2 / L 1=3 / 9=\frac{1}{3}$. Can gravitational lensing explain the time structure and brightness ratio?

From the Kamiokande pulse brightness ratio $L_{2} / L_{1}=\frac{1}{3}$ one computes $\alpha_{0}=3.59 \beta$. From the value of the time delay of $\Delta t=9.219$ and $\frac{\alpha_{0}}{\beta}$ one calculates the lens mass at about 414,000 solar masses. A scan of the lens distance from 1 to $49 \mathrm{kpc}$ gives an angular separation between the two images, $\alpha$ from 3.8 arc seconds at the shortest distance to 0.08 at $49 \mathrm{kpc}$. The lens source angular separation $(\beta)$ as a function of the distance to the lens is shown in Figure 3. It drops from 1.02 arc seconds near the observer to 0.03 to 0.02 near the source.

Comparison with other experiments is difficult since Kamiokande did not have a synchronized clock. Baksan had a synchronized signal but it was about 30.448 after the synchronized IMB signal. This Baksan time shift will be revisited in the next section. One can analyze the Baksan signal itself for compatibility with the lensing hypothesis.

The Baksan signal of five events has a structure of three early events over $1.71 \mathrm{~s}$ followed by two events in $1.412 \mathrm{~s}$, Figure 2. The gap between these bunches is $7.687 \mathrm{~s}$. The ratio of the energy in each pulse is, crudely, the ratio of the number of events, which is $0.667 \pm 0.609$ which is not in conflict with the Kamiokande observations. Thus, Baksan may have seen the first pulse at 1.3 to 1.5 times the nominal brightness and a second pulse suppressed to 0.3 to 0.5 the nominal brightness. While not a strong confirmation since the brightness ratio is very weak, the Baksan results support the two pulse gravitational lens interpretation.

IMB also had a time structure in its event sample. Of the eight events observed overall, five occurred in the first 1.57 seconds of the burst, compatible with the time scale of the first nine Kamiokande events. The last two IMB events occurred in $0.58 \mathrm{~s} 5.01 \mathrm{~s}$ after the initial event. The 6 'th IMB event occurred at $2.69 \mathrm{~s}$ which is outside the $2 \mathrm{~s}$ duration of 
the "first" pulse observed at Kamiokande and Baksan. The ratio of late to early events, $2 / 6$, is compatible with the other evidence. The second pulse seems to be too early to fit the lensing picture supported by the other observations. The shorter time gap suggests a smaller lensing mass but also casts doubt on the lens hypothesis.
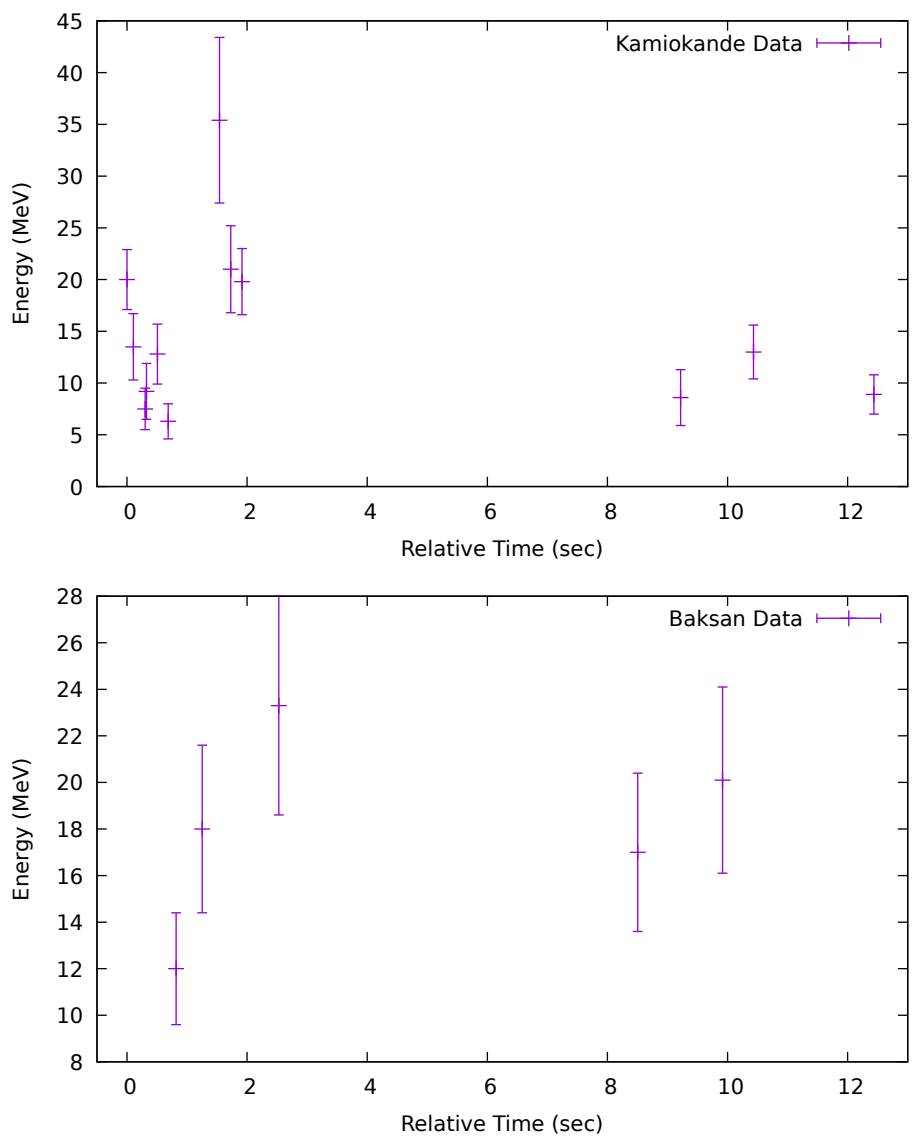

Figure 2. The Kamiokande neutrino events (top) and Baksan (bottom) from SN1987A plotted as event energy as a function of relative time. There is a notable gap in both data samples. The relative time between the plots is arbitrary. It is not the nominal time listed in Table 1.

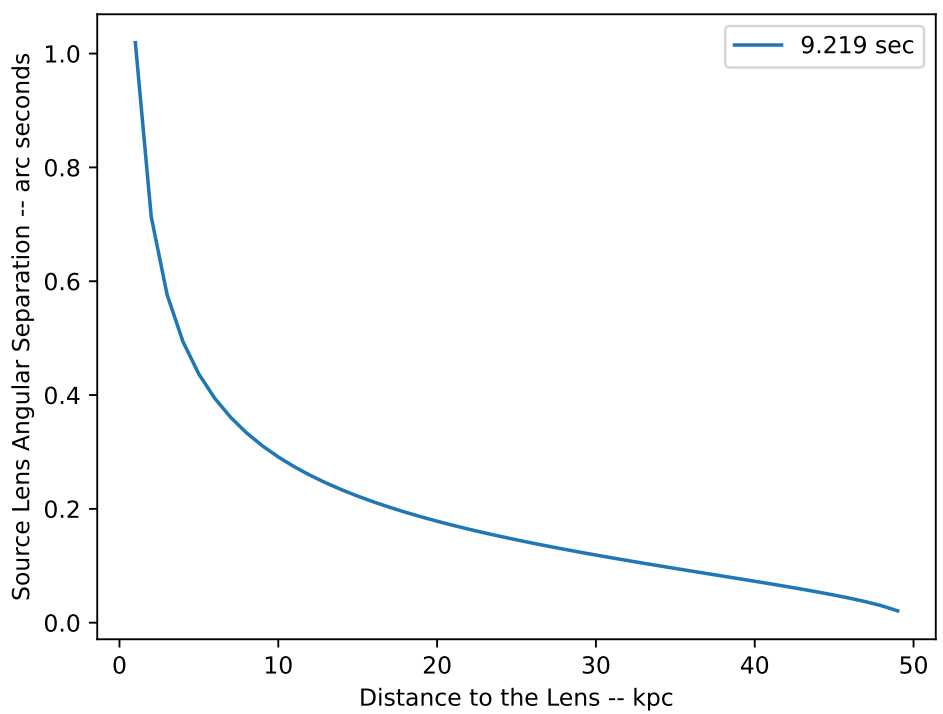

Figure 3. The angle between the lens and the source, $\beta$, in seconds of arc versus the distance to the lens for a solution for $M$ and $\alpha_{0} / \beta$ that fits the Kamiokande time and brightness structure. 
There is no known star cluster in the direction of the LMC to provide the mass. The source lens alignment, Figure 3 seems rather unlikely. The standard estimate [18] of the galactic "optical depth" for lensing a source in the LMC is about $10^{-6}$ independent of the lensing mass.

In terms of the physics of supernovae the lensing amplified the energy recorded by Kamiokande by a factor of $\frac{L_{1}+L_{2}}{L_{1}-L_{2}} \approx 2.0$, since the first pulse is brightened by a factor of 1.5 and the second pulse adds in another 0.5. Thus, the reported energy in electron antineutrinos of $8 \times 10^{52} \mathrm{ergs}[10,11]$ is an over estimate.

\section{Alternate Explanations}

A number of ideas have been put forward to explain the UNO event time. While UNO did not report a signal at the 7:35:41 time reported for other neutrino observations, they have reported coincidences with a gravitational wave bar detector [19]. The absence of a neutrino signal in UNO at 7:35:41 is not unexpected since its mass is small, 2.2 times smaller than Baksan, so it would be expected to have only 2.25 events at that time.

It has been suggested that the $30 \mathrm{~s}$ delay of Baksan with respect to other observations is due to a systematic $30 \mathrm{~s}$ advance added to the time standard in the USSR [20].

Oyama $[21,22]$ has suggested that the $7.3 \mathrm{~s}$ gap in the Kamiokande data is due to an instrumentation inefficiency that made data collection impossible in that interval.

The most popular opinion is that UNO is unexplained but not identified with SN1987A and that the time structures and energy differences of the other observations are due to instrumental differences and statistical fluctuations in small data samples. The late time distribution of neutrino events is considered indicative of neutrino cooling of the source.

\section{Gravitational Lensing of Optical Supernovae}

Gravitational lensings of the optical components of supernovae have been seen [23]. Four images of a supernova at $z=1.49$ were created by a lensing galaxy, J1149.5 +2223 , at $z=0.54$. The lensing manifested itself as four images observed in April 2015. A fifth image appeared in December 2015 at a predicted location.

Goobar et al. [24] reported multiple images from a Type 1a supernova at $z=0.409$ at an amplification of more than 50 , lensed by a galaxy at $z=0.216$. Four images were resolved.

Several additional supernovae have been lensed [25-30], manifesting higher brightness than expected.

\section{Conclusions}

Gravitational lensing can distort the observation of any distant object including a supernova neutrino burst. It is unlikely but should not be overlooked if the data shows noteworthy departures from expectations such as multiple pulses or unexpectedly large signals. Redundancy of observations is helpful in avoiding misleading results from statistical fluctuations.

The distribution of neutrinos from SN1987A have a time and brightness structure expected from a lensing event. The mass and angular alignment make this unlikely but perhaps optical confirmation of such a lens is still possible. A lens will influence starlight coming from behind it if the alignment is appropriate. Observing a time delay as suggested by the neutrino burst requires some time structure in the source of tens of seconds or less. It also requires detecting equipment sensitive to short time scale variation in the brightness. On the other hand, an optical detection may be able to measure the angular separation of the images and the ratio of their brightness. It is likely that alignments have changed over the decades and one must search back through the historical record. Multiple images of a single star in the LMC are hard to distinguish from multiple stars in the LMC, especially since the images will not have the same apparent luminosity. Lensing of starlight is difficult since, unless the alignment with the lens changes, there is no way to tell that the image has been displaced from its flat space location. Image distortion or brightness variation caused by microlensing would help to identify the presence of a lens. 
The lensing hypothesis for the observed SN1987A neutrino signal would reduce the total energy estimate derived from the observation since the brightness would have been enhanced. On the other hand the average neutrino power in the pulse would increase since the duration of the pulse would be shortened to 2-3 s. Not all observations support this interpretation.

Funding: This research received no external funding

Acknowledgments: I am grateful to Kate Scholberg who convinced me that one need not consider SN1987A as typical. John Learned, Bob Svoboda and Ralph Becker-Szendy have contributed important ideas to a discussion initiated by reference [21]. Erica Caden filled me in on the status of direction finding with the $\bar{v}_{e} P$ reaction, reference [17]. Jim Rich had many suggestions after reviewing an early version of the manuscript, including updating to a more modern notation.

Conflicts of Interest: The author declares no conflict of interest.

\section{References}

1. Longo, M.J. New Precision Tests of the Einstein Equivalence Principle from Sn1987a. Phys. Rev. Lett. 1988, 60, 173-175. [CrossRef]

2. Krauss, L.M.; Tremaine, S. Test of the Weak Equivalence Principle for Neutrinos and Photons. Phys. Rev. Lett. 1988, 60, 176-177. [CrossRef]

3. Shapiro, I.I. Fourth Test of General Relativity. Phys. Rev. Lett. 1964, 13, 789. [CrossRef]

4. LoSecco, J.M. Limits on CP invariance in general relativity. Phys. Rev. D 1988, 38, 3313. [CrossRef] [PubMed]

5. Pakvasa, S.; Simmons, W.A.; Weiler, T.J. Test of equivalence principle for neutrinos and antineutrinos. Phys. Rev. D 1989, $39,1761$. [CrossRef] [PubMed]

6. Klimov, Y.G. Deflection of light rays in the gravitational fields of galaxies. Dokl. Akad. Nauk SSSR 1963, 148, 789-792.

7. Refsdal, S.; Bondi, H. The Gravitational Lens Effect. Mon. Not. R. Astron. Soc. 1964, 128, 295. [CrossRef]

8. Liebes, S. Gravitational Lenses. Phys. Rev. 1964, 133, B835. [CrossRef]

9. Refsdal, S. On the Possibility of Determining Hubble's Parameter and the masses of Galaxies from the Gravitational Lens Effect. Mon. Not. R. Astron. Soc. 1964, 128, 307. [CrossRef]

10. Hirata, K.; Kajita, T.; Koshiba, M.; Nakahata, M.; Oyama, Y.; Sato, N.; Suzuki, A.; Takita, M.; Totsuka, Y.; Kifune, T.; et al. [Kamiokande-II]. Observation of a neutrino burst from the supernova SN1987A. Phys. Rev. Lett. 1987, 58, 1490. [CrossRef]

11. Hirata, K.S.; Kajita, T.; Koshiba, M.; Nakahata, M.; Oyama, Y.; Sato, N.; Suzuki, A.; Takita, M.; Totsuka, Y.; Kifune, T.; et al. Observation in the Kamiokande-II detector of a neutrino burst from the supernova SN1987A. Phys. Rev. D 1988, 38, 448-458. [CrossRef] [PubMed]

12. Bionta, R.M.; Blewitt, G.; Bratton, C.B.; Casper, D.; Ciocio, A.; Claus, R.; Cortez, B.; Crouch, M.; Dye, S.T.; Errede, S.; et al. Observation of a neutrino burst in coincidence with supernova 1987A in the Large Magellanic Cloud. Phys. Rev. Lett. 1987, 58, 1494. [CrossRef] [PubMed]

13. Alekseev, E.N.; Alekseeva, L.N.; Krivosheina, I.V.; Volchenko, V.I. Properties of the Supernova 1987A Neutrino Signal Recorded by the Baksan Underground Scintillation Telescope. Sov. Astron. Lett. 1988, 14, 41.

14. Alekseev, E.N.; Alekseeva, L.N.; Krivosheina, I.V.; Volchenko, V.I. Detection of the neutrino signal from SN 1987A using the INR Baksan underground scintillation telescope. In Proceedings of the ESO Workshop on the SN 1987A, Garching, Germany, 6-8 July 1987; Proceedings (A88-35301 14-90); European Southern Observatory; pp. 237-247; discussion p. 247.

15. Alekseev, E.N. Detection of the neutrino signal from SN 1987A using the INR Baksan underground scintillation telescope. Lett. JETPh (Sov.) 1987, 45, 589, (461 in the original Russian).

16. Aglietta, M.; Badino, G.; Bologna, G.; Castagnoli, C.; Castellina, A.; Dadykin, V.L.; Fulgione, W.; Galeotti, P.; Kalchukov, F.F.; Kortchaguin, B.; et al. On the Event Observed in the Mont Blanc Underground Neutrino Observatory during the Occurrence of Supernova 1987a. EPL (Europhys. Lett.) 1987, 3, 1315. [CrossRef]

17. Caden, E. Studying Neutrino Directionality with the Double Chooz Experiment. Ph.D. Thesis, Drexel University, Philadelphia, PA, USA, June 2013. Available online: https:/ / idea.library.drexel.edu/islandora/object/idea:4167 (accessed on 13 July 2021).

18. Griest, K. Galactic Microlensing as a Method of Detecting Massive Compact Halo Objects. Astrophys. J. 1991, 366, 412. [CrossRef]

19. Amaldi, E.; Bonifazi, P.; Castellano, M.G.; Coccia, E.; Cosmelli, C.; Frasca, S.; Gabellieri, M.; Modena, I.; Pallottino, G.V.; Pizzella, G.; et al. Data Recorded by the Rome Room Temperature Gravitational Wave Antenna, during the Supernova SN 1987a in the Large Magellanic Cloud. Europhys. Lett. 1987, 3, 1325. [CrossRef]

20. J. Learned private communication, email 16 May 2021.

21. Yuichi Oyama. About 30 Years from Kamiokande. 22 April 2021. from the Author. Available online: https://www-nu.kek.jp/ oyama/kam30yrs.oyama.210422.pptx (accessed on 6 May 2021).

22. Oyama, Y. Comment on 'Observation of a Neutrino Burst from the Supernova SN1987A'. arXiv 2021, arXiv:2108.01783

23. Kelly, P.L.; Rodney, S.A.; Treu, T.; Strolger, L.G.; Foley, R.J.; Jha, S.W.; Selsing, J.; Brammer, G.; Bradač, M.; Cenko, S.B.; et al. Déjà $\mathrm{Vu}$ All Over Again: The Reappearance of Supernova Refsdal. arXiv 2016, arXiv:1512.04654v1. 
24. Goobar, A.; Amanullah, R.; Kulkarni, S.R.; Nugent, P.E.; Johansson, J.; Steidel, C.; Law, D.; Mörtsell, E.; Quimby, R.; Blagorodnova, N.; et al. iPTF16geu: A multiply imaged, gravitationally lensed type Ia supernova. Science 2017, 356, 291-295. [CrossRef]

25. Goobar, A.; Paech, K.; Stanishev, V.; Amanullah, R.; Dahlén, T.; Jönsson, J.; Kneib, J.P.; Lidman, C.; Limousin, M.; Mörtsell, E.; et al. Near-IR search for lensed supernovae behind galaxy clusters. Astron. Astrophys. 2009, 507, 71. [CrossRef]

26. Patel, B.; McCully, C.; Jha, S.W.; Rodney, S.A.; Jones, D.O.; Graur, O.; Merten, J.; Zitrin, A.; Riess, A.G.; Matheson, T.S.; et al. Three Gravitationally Lensed Supernovae behind CLASH Galaxy Clusters. Astrophys. J. 2014, 786, 9. [CrossRef]

27. Rodney, S.A.; Strolger, L.G.; Kelly, P.L.; Bradač, M.; Brammer, G.; Filippenko, A.V.; Foley, R.J.; Graur, O.; Hjorth, J.; Jha, S.W.; et al. SN Refsdal: Photometry and Time Delay Measurements of the First Einstein Cross Supernova. Astrophys. J. 2016, 811, 70. [CrossRef]

28. Chornock, R.; Berger, E.; Rest, A.; Milisavljevic, D.; Lunnan, R.; Foley, R.J.; Soderberg, A.M.; Smartt, S.J.; Burgasser, A.J.; Challis, P.; et al. PS1-10afx at $z=1.388$ : Pan-STARRS1 Discovery of a New Type of Superluminous Supernova. Astrophys. J. 2013, 767, 162. [CrossRef]

29. Quimby, R.M.; Oguri, M.; More, A.; More, S.; Moriya, T.J.; Werner, M.C.; Tanaka, M.; Folatelli, G.; Bersten, M.C.; Maeda, K.; et al. Detection of the Gravitational Lens Magnifying a Type Ia Supernova. Science 2014, 344, 396. [CrossRef]

30. Quimby, R.M.; Werner, M.C.; Oguri, M.; More, S.; More, A.; Tanaka, M.; Nomoto, K.I.; Moriya, T.J.; Folatelli, G.; Maeda, K.; et al. Extraordinary Magnification of the Ordinary Type Ia Supernova PS1-10afx. Astrophys. J. Lett. 2013, 768, L20. [CrossRef] 